\title{
Predictive Neural Model of an Osmotic Dehydration Process
}

\author{
Ieroham Baruch,' Próspero Genina-Soto ${ }^{2}$ and Josefma Barrera-Cortes ${ }^{2}$ \\ 'Department of Automatic Control and ${ }^{2}$ Department of Biotechnology and \\ Bioengineering, Ave. IPN.No 2508, A.P. 14-470, 07360 Mexico D.F., Mexico
}

\begin{abstract}
This paper proposed the use of a Recurrent Neural Network model (RNN) for predicting the osmotic dehydration kinetics of nature product cubes (apples, sweet potatoes, potatoes) at different operational conditions of temperature and concentration of the osmotic solution. The proposed RNN model has five inputs, three outputs, and eight neurons in the hidden layer, with global and local feedbacks. The learning algorithm is a modified version of the dynamic backpropagation algorithm. The learning and generalization errors are below $2 \%$. Learning was performed in 701 epochs of 40 iterations each. The statistical analysis confirmed the good quality of the proposed RNN model.
\end{abstract}

\section{KEYWORDS}

process prediction, recurrent neural network model, backpropagation learning, statistical analysis, biotechnological process, osmotic dehydration kinetics of fruit cubes.

\section{INTRODUCTION}

Osmotic Dehydration (OD) is a mass transfer operation of increasing interest in food preservation technologies. The operation is carried out by

Reprint requests to: Ieroham Baruch, Department of Automatic Control, Ave. IPN No 2508, A.P. 14-470, 07360 Mexico D.F., Mexico; e-mail: baruch@ ctrl.cinvestav.mx 
immersing a nature product in a hypertonic solution for achieving a flow of water outward from the solid, as well as a countercurrent flow of solution into the product. For developing a food treatment technology based on the OD process, it is necessary to know the OD kinetics for fixing the desired dehydration and impregnation levels involved in such process (see RaoultWack, 1994).

For modeling mass transfer phenomena during the OD process, analytical and empirical models have been reported. Several empirical models are those developed by Lenart \& Flink (1984) and Azuara et al. (1998). Analytical models have been developed by Beristain et al. (1992), who proposed a phenomenological model based on Fick's second law. The authors (RaoultWack et al., 1991) developed a compartmental model, and Toupin et al. (1989) stated a complex mathematical model based on transport phenomena. An inconvenience of most models is that they involve certain empirical constants that must be known previously to be used.

Artificial Neural Networks (ANN) models seemed suitable for modeling the osmotic dehydration process (see Bishop, 1994). Artificial Neural Networks have been applied in various fields since 1985 and are beneficial for modeling complicated and atypical experimental conditions, without having to put forward hypotheses on the underlying physical and/or chemical mechanisms (see Baughman \& Liu, 1995). Such models also take nonlinearities and interactions between input variables of the model into account with very few parameters (see Valdez-Castro et al., 2003).

Our study was designed to test and validate the efficiency of Recurrent Neural Networks (RNN) for predicting the profiles kinetics of water loss in such fruits as sweet potatoes, potatoes, and apples. The kinetics profile is determined at two temperatures $\left(26^{\circ} \mathrm{C}\right.$ and $\left.50^{\circ} \mathrm{C}\right)$ and three concentrations of the osmotic solution $(30: 100,50: 100,70: 100)$ (see Genina-Soto et al., 2001).

\section{RECURRENT NEURAL NETWORK TOPOLOGY AND LEARNING}

In the present paper, a two-layer, completely parallel RNN model is proposed to be used as a neural predictive model of the OD process. The 
RNN two-layer architecture contains one hidden and one output layer, both recurrent-with local and global feedbacks. The proposed RNN model is inspired from the RNN model given in Baruch et al. (2002) and Nava et al. (2004), but here we extended it by a global feedback and by a local feedback in the output layer, so that it takes the form:

$$
\begin{aligned}
& X(k+1)=S\left[A_{1} X(k)+B U(k)+D Y(k)\right] \\
& Y(k+1)=S\left[C X(k)+A_{2}(k) Y(k)\right] \\
& A_{1}=\text { block-diag }\left(A_{1, i j}\right) ;\left|A_{1, j i j}\right|<1 ; A_{2}=\text { block-diag }\left(A_{2, i j}\right) ;\left|A_{2, j \mathrm{j}}\right|<1,
\end{aligned}
$$

where:

$\mathrm{Y}, \mathrm{X}$, and $\mathrm{U}$ are output, state, and input vectors, with dimensions $1, \mathrm{n}, \mathrm{m}$, respectively;

$A_{1}, A_{2}$ are (nxn) and (lxI) - local feedback block-diagonal weight matrices;

$A_{1, i j}, A_{2, i j}$ are diagonal blocks of $A_{1}, A_{2}$ with ( $\left.1 \times 1\right)$ or (2x2) dimensions;

$B$ and $C$ are (nxm) and (lxn) - weight matrices;

$\mathrm{D}$ is a (nxI) - global output closed loop matrix; and

$\mathrm{S}[$.$] is a vector-valued sigmoid activation function.$

Equation (3) represents the local stability condition imposed on all diagonal blocks of the feedback matrices $A_{1}, A_{2}$. The saturation function could be used as approximation of the sigmoid function to improve the RNN architecture, facilitating its realization. The stability of the RNN model is assured by the activation functions $S$ and by the local stability condition, given by Eq. (3). The global feedback has been introduced to reduce the measurement data noise during the RNN learning. For training the RNN model, a modified dynamic backpropagation algorithm is used (Baruch et al., 2002; Nava et al., 2004), which has the form:

$$
W_{i j}(k+1)=W_{i j}(k)+\eta \Delta W_{i j}(k)+\alpha \Delta W_{i j}(k-1)
$$

where: 
$\mathrm{W}_{\mathrm{ij}}$ is a general weight, denoting the $\mathrm{ij}$-th weight element of each weight matrix $\left(C, D, A_{1}, A_{2}, B\right)$ in the RNN model to be updated;

$\Delta \mathrm{W}_{\mathrm{ij}}\left(\Delta \mathrm{C}_{\mathrm{ij}}, \Delta \mathrm{D}_{\mathrm{ij}}, \Delta \mathrm{A}_{1 \mathrm{ij}}, \Delta \mathrm{A}_{2 \mathrm{ij}}\right.$ and $\left.\Delta \mathrm{B}_{\mathrm{ij}}\right)$ is the weight correction of $\mathrm{W}_{\mathrm{ij}}$; and finally,

$\eta$, and $\alpha$ are learning and momentum rate parameters.

The stability of this learning algorithm was proved in Baruch et al. (2002) and Nava et al. (2004). In the discrete-time RNN model, the weight corrections of the updated matrices, described by Eqs. (1) and (2), given as follows:

- For the output layer:

$$
\begin{aligned}
& R_{1}=\left[T_{j}(k)-Y_{j}(k)\right] Y_{j}(k)\left[1-Y_{j}(k)\right] \\
& \Delta C_{i j}(k)=R_{1} X_{i}(k) \\
& \Delta A_{2, i j}(k)=R_{1} Y_{i}(k)
\end{aligned}
$$

where:

$\Delta \mathrm{C}_{\mathrm{ij}}, \Delta \mathrm{A}_{2, \mathrm{jj}}$ are weight corrections of the $\mathrm{ij}$-th elements of the (lxn) learned matrix $\mathrm{C}$ and the ( $\mathrm{xl}$ ) learning matrix $\mathrm{A}_{2}$;

$T_{j}$ is $a_{j} j$-th element of the target vector,

$Y_{j}$ is a j-th element of the output vector,

$X_{i}$ is an $i-t h$ element of the output vector of the hidden layer,

and

$R_{1}$ is an auxiliary variable.

- For the hidden layer:

$$
\begin{aligned}
& R_{2}=C_{1}(k)[T(k)-Y(k)] X_{j}(k)\left[1-X_{j}(k)\right] \\
& \Delta B_{i j}(k)=R_{2} U_{i}(k) \\
& \Delta D_{i j}(k)=R_{2} Y_{i}(k)
\end{aligned}
$$




$$
\Delta A_{1, j j}(k)=R_{2} X_{i}(k-1)
$$

where:

$\Delta B_{i j}, \Delta D_{i j}$, are weight corrections of the ij-th elements of the (nxm) learned matrix $B$ and the (nxl) learned matrix $D$;

$C_{i}$ is a row vector of dimension $(|x|)$, taken from the transposed matrix $\mathrm{C}^{\prime}$;

[T-Y] is a (lxl) output error vector, through which the error is backpropagated to the hidden layer,

$U_{i}$ is an $i$-th element of the input vector $U$;

$\mathrm{X}_{\mathrm{i}}$ is an $\mathrm{i}$-th element of the vector $\mathrm{X}$;

$\Delta A_{1 . j j}$ is the weight correction of the $i j-t h$ elements of the (nxn) blockdiagonal matrix $\mathrm{A}_{1}$; and

$R_{2}$ is an auxiliary variable.

\section{OSMOTIC DEHYDRATION PROCESS EXPERIMENT DESCRIPTION}

The nature products (sweet potatoes, potatoes, apples) were obtained from a local market. The osmotic solutions were prepared using distilled water and commercial refined sucrose as osmotic agent.

The Osmotic Dehydration (OD) process experiment requires following a sequence of operation. Nature product cubes of $3.5 \mathrm{~cm}$. are put into a container (4L) containing osmotic solutions at a fixed concentration and temperature. The homogenization of the osmotic solution is achieved by mildly agitating at 100 rpm (Genina-Soto et al., 2001). The osmotic dehydration process is treated with osmotic solutions of different sucrose concentrations (30:100, 50:100, 70:100 $\mathrm{w} / \mathrm{w})$ and temperature $\left(26^{\circ} \mathrm{C}\right.$ and $\left.50^{\circ} \mathrm{C}\right)$. The osmotic dehydration process is carried out during an exposition time of 168 hours. During this time, the nature cubes are tested at $8.24,72$, and 168 hours.

The global OD kinetics is obtained by the weight evolution of the cubes. The cubes are withdrawn from the solution, immediately blotted with a tissue paper, and weighted on tarred receptacles before returning them to the reactor. The water lost is determined by the weight difference between dried and osmosis cubes. 


\section{INPUT-OUTPUT PATTERN OF LEARNING}

The variables determining the OD process are the temperature $(T)$ and the osmotic solution concentration (OSC). The RNN model includes these variables in the input pattern of learning, as well as the dimensional loss of water $(\mathrm{C} / \mathrm{Co})$ for the three fruits studied: sweet potatoes, potatoes, and apple. The output pattern of learning is conformed only by the dimensional loss of water $(\mathrm{C} / \mathrm{Co})$ of the three fruits mentioned below and corresponding at a next time of the OD process (see Fig. 1 for details).

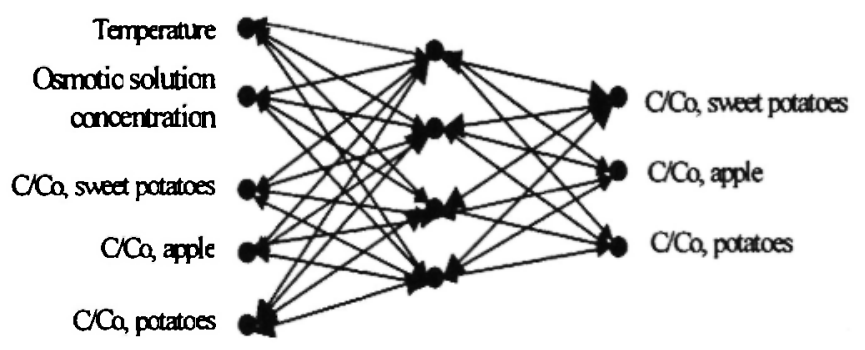

Fig. 1: Input-output pattern of learning.

\section{RESULTS}

The results for the last epoch of learning and generalization are given graphically in Fig. 2 and Fig. 3, respectively. The RNN used for modeling and identification of the OD process has five inputs, eight neurons in the hidden layer and three outputs $(3,5,8)$. The number of neurons in the hidden layer is determined in an experimental way, according to the Mean Square Error (MSE\%) of learning. The learning algorithm is a version of the dynamic backpropagation algorithm especially designed for this RNN topology. The learning algorithm described above is applied simultaneously to 5 OD kinetic data sets containing 40 points ( 8 points each).

The learning rate is $\eta=1.2$ and the momentum rate is $\alpha=0.2$. The five data sets are considered as an epoch of learning. After each epoch of learning, the five OD kinetic data sets are interchanged in an arbitrary manner from one 
epoch to another. An unknown kinetic data set repeated five times is used as a generalization data set. The learning is stopped when the MSE\% of learning becomes below of a prescribed error limit (MSE\% $<2 \%$ ). The same is done for the generalization, where $\mathrm{MSE} \%<2 \%$. An additional measure of weight convergence was also applied, which is the relation $|\Delta \mathrm{Wij}(\mathrm{k})| /\left.\mathrm{Wij}(\mathrm{k})\right|^{*} 100 \%$ $<2 \%$ for all updated parameters. This error was obtained after 701 epochs of learning.
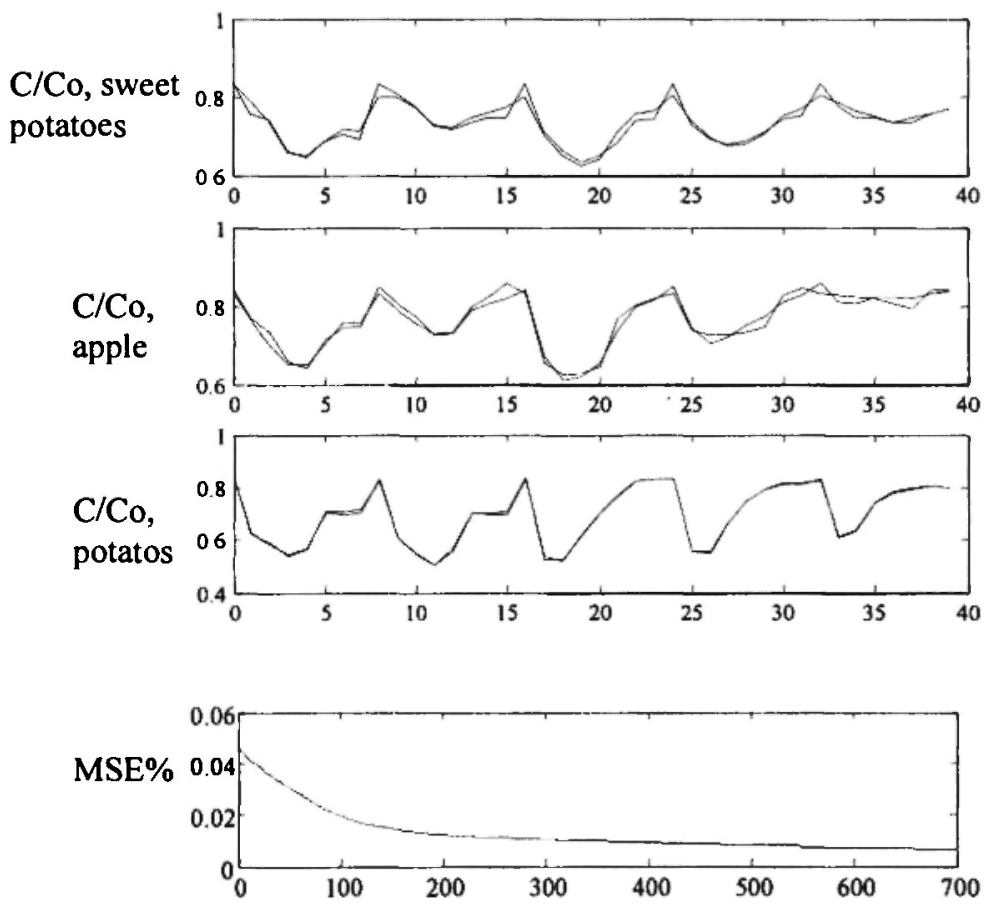

Fig. 2: Learning results: Experimental data (continuous line) vs. RNN outputs (dashed line) are plotted subsequently for the last epoch of learning. The variables shown are relative residual water content of the natural fruits: sweet potatoes, potatoes, and apple, as well as the mean square error of learning during 701 epochs of learning. 
The results, shown graphically in Fig. 2, compare the experimental data for the five OD kinetics studied. The variables compared are the relative residual water content of each fruit tested and the mean square error reached after 701 epochs of learning. The generalization of the RTNN was carried out reproducing $\mathrm{OD}$ kinetics, which is not included in the training process. This OD process was carried out at $20^{\circ} \mathrm{C}$ and an osmotic solution concentration of 30:100. The generalization results, shown in graphic form in Fig. 3, compare the experimental data with the output data, generated by the RNN during the
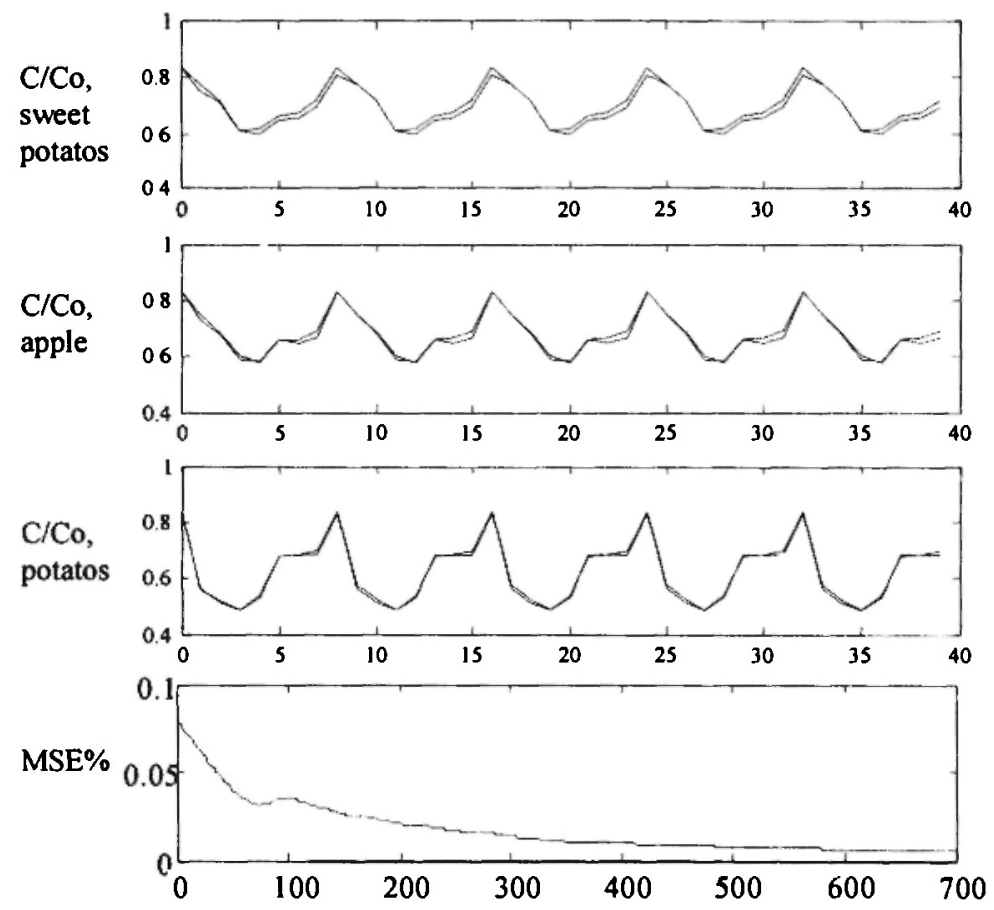

Fig. 3: Generalization results: Experimental data (continuous line) vs. RNN outputs (dashed line) are plotted for the last epoch of generalization. The variables shown are relative residual water content of natural sweet potatoes, potatoes, and apple, as well as the mean square error of generalization during 701 epochs of generalization. 
TABLE 1

Means squared error (\%) in the end of the 701-th epoch of learning/ generalization for 20 runs of the program.

\begin{tabular}{|c|c|c|c|c|c|}
\hline No. & 1 & 2 & 3 & 4 & 5 \\
\hline Learning & 0.68 & 0.63 & 0.73 & 0.76 & 0.75 \\
\hline Generalization & 0.69 & 0.67 & 0.72 & 0.92 & 0.88 \\
\hline No. & 6 & 7 & 8 & 9 & 10 \\
\hline Learning & 0.68 & 0.71 & 0.72 & 0.65 & 0.68 \\
\hline Generalization & 0.69 & 0.65 & 0.79 & 0.67 & 0.69 \\
\hline No. & 11 & 12 & 13 & 14 & 15 \\
\hline Learning & 0.73 & 0.75 & 0.63 & 0.76 & 0.63 \\
\hline Generalization & 0.72 & 0.88 & 0.67 & 0.92 & 0.67 \\
\hline No. & 16 & 17 & 18 & 19 & 20 \\
\hline Learning & 0.76 & 0.68 & 0.73 & 0.75 & 0.71 \\
\hline Generalization & 0.92 & 0.69 & 0.72 & 0.88 & 0.65 \\
\hline
\end{tabular}

last epoch of generalization. The MSE\% of generalization, given on the end of the last graphic is below $2 \%$. The MSE\% of 20 runs of the program at the end of the 701-th epoch of learning/generalization is given in Table 1.

The operational conditions of this $O D$ process are in the range of the operational conditions studied. According to the results obtained, the capacity of the RNN to reproduce similar degradation process, entering with an incomplete or noisy input data set is confirmed.

The cost function $\xi$ minimized during the learning is the average of the instantaneous error $e_{i}$ of the output layer, given by the following equation:

$$
\xi=\frac{1}{2} \sum_{i} e_{i}^{2}
$$

where $i$ is the number of outputs shown in Figs. 2 and 3, which is equal to three. The average cost $\xi_{\mathrm{av}}$ for one epoch of learning is: 


$$
\xi_{a v}=\frac{1}{i v} \sum_{j=1}^{N} \xi_{j}
$$

where $\mathrm{j}$ is an iteration number and $\mathrm{N}$ is the total number of iterations in one epoch, equal to 40 . The average cost for all epochs $\xi_{\mathrm{av}, \mathrm{ep}}$ of learning is:

$$
\xi_{a v, e p}=\frac{1}{N e p} \sum_{j=1}^{N e p} \xi_{a v_{j}}
$$

where $\mathbf{j}$ is the epoch number and $\mathrm{N}_{\mathrm{ep}}$ is the total number of learning epochs, equal to 701 . The mean average cost for all runs $\varepsilon$ of learning is:

$$
\varepsilon-\frac{1}{n} \sum_{k=1}^{n} \xi_{a v_{k}}
$$

where $\mathrm{k}$ is the run number and $\mathrm{n}$ is the total number of runs, equal to 20 . The standard deviation $\sigma$ with respect to the mean value is:

$$
\sigma=\sqrt{\frac{1}{n} \sum_{i=1}^{n} \Delta^{2}}
$$

where the deviation $\Delta$ is:

$$
\Delta=\xi_{\mathrm{av}}-\varepsilon
$$

The results for the statistical data in Table 1 are depicted in graphic form, in Fig. 4 for learning and in Fig. 5 for generalization. The mean and the standard deviation values are respectively:

For learning: $\varepsilon=0.706 \% ; \sigma=0.045 \%$.

For generalization: $\varepsilon=0.754 \% ; \sigma=0.103 \%$.

Figures 4 and 5 show that about two or three run data are outside the standard deviation, which is due to the different initial weights values, chosen in an arbitrary manner as equiprobable numbers over a small range.

Importantly, the experimental data in the OD process have an average standard deviation of $\pm(2-10) \%$. According to the mean square error of 
approximation obtained during RNN model learning, one could say that the RNN model could be used successively to predict the OD process.

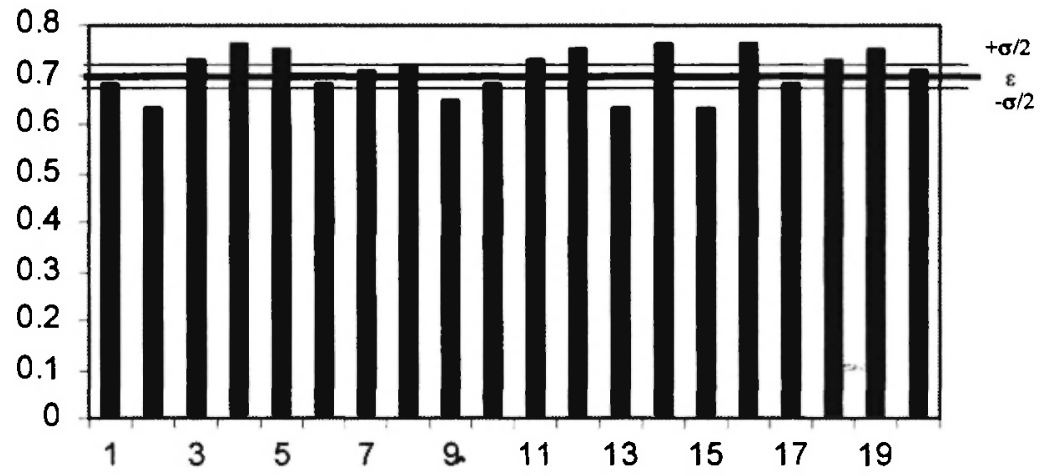

Fig. 4: Graphic representation of the Mean squared error (\%) in the end of the 701th epoch of learning for 20 runs of the program, together with the mean value and the standard deviation

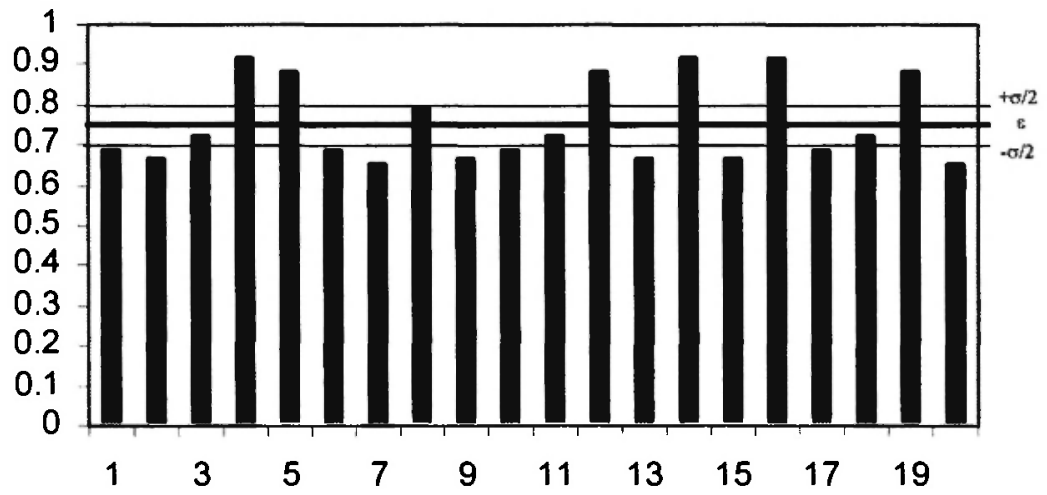

Fig. 5: Graphic representation of the Mean squared error (\%) in the end of the 701th epoch of generalization for 20 runs of the program, together with the mean value and the standard deviation. 


\section{CONCLUSIONS}

This paper proposes to use a Recurrent Neural Network model to predict the OD process. The multi-input multi-output RNN proposed has five inputs, three outputs, and eight neurons in the hidden layer, as well as global and local feedbacks. The weight-update-learning algorithm is a version of the well-known dynamic backpropagation algorithm, directed to RNN learning. The learning and generalization error obtained at the end of the 701 -th epoch is below $2 \%$. The learning process is applied simultaneously for five OD kinetics data of different temperature, different osmotic solution concentration, and different relative residual water content of the natural fruits like sweet potatoes, potatoes, and apple, for which good convergent results, including statistics, were obtained. After training and generalization, the RNN model is capable of evolving the relative residual water content of the natural fruits like sweet potatoes, potatoes, and apple, entering with an incomplete or noisy input data set. The last statistical results confirm the good work of the learning and generalization programs, which give similar results in various runs.

\section{REFERENCES}

Azuara, E., B eristain, C.I. and Gutierrez, G.F. 1988. A method for continuous kinetic evaluation of osmotic dehydration. Lebensmittel - Wissenschast \& Technologie, 31, 317-321.

Baruch, I., Flores, J.M., Nava, F., Ramirez, I. R. and Nenkova, B. 2002. An advanced neural network topology and learning, applied for identification and control of a D.C. motor. Proceedings of the First International IEEE Symposium on Intelligent Systems, IS'02, Varna, Bulgaria, I, 289-295.

Baughman, D.R. and Liu, Y.A. 1995. Neural networks in bioprocessing and chemical engineering. New York, NY, USA, Academic Press, 1-17.

Beristain, C.I., Azuara, E. and García, H. S. 1992. Development of a mathematical model to predict kinetics of osmotic dehydration. Journal of Food Science and Technology, 29, 239-242.

Bishop, C.M. 1994. Neural networks and their applications. Review of Scientific Instruments, 65, 1803-1832.

Genina-Soto, P., Barrera-Cortés, J., Gutiérrez-López, G. and Nieto, E.A. 2001. Temperature and concentration effects of osmotic media on OD 
profiles of sweet potato cubes. International Journal of Drying Technology, $19,547-558$.

Lenart, A. and Flink, J.M. 1984. Osmotic concentration of potatoes. II. Spatial distribution of the osmotic effect. Journal of Food Technology, $19,65-89$.

Nava, F., Baruch, I., Pozniak, A. and Nenkova B. 2004. Stability proofs of advanced recurrent neural network topology and learning. Comptes Rendus (Proceedings of the Bulgarian Academy of Sciences), 57, 27-32. ISSN 0861-1459.

Raoult-Wack, A.L., Petitdemange, F., Giroux, F., Guilbert, S., Rios, G. and Lebert, A. 1991. Simultaneous water and solute transport in shrinking media, Part 2, A compartmental model for dewatering and impregnation soaking process. International Journal of Drying Technology, 9, 613-630.

Raoult-Wack, A.L. 1994. Recent advances in the osmotic dehydration of foods. Trends in Food Science and Technology, 5, 255-260.

Toupin, C.J., Marcotte, M. and Le Maguer, M. 1989. Osmotically-induced mass transfer in plant storage tissues, a mathematical model, Part I. Journal of Food Engineering, 10, 13-38.

Valdez-Castro, L., Baruch, I. and Barrera-Cortés, J. 2003. Neural networks applied to the prediction of fed-batch fermentation kinetics of Bacillus thuringiensis. Bioprocess and Biosystems Engineering, 25, 229233. 
